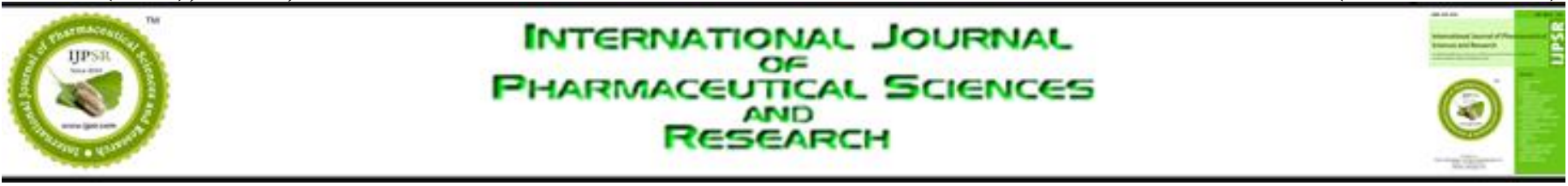

Received on 30 March, 2013; received in revised form, 09 May, 2013; accepted, 15 July, 2013; published, 01 August, 2013

\title{
FLOATING DRUG DELIVERY SYSTEM: A REVIEW
}

Juhi Dubey* and Navneet Verma

Department of Pharmaceutics, IFTM University, Moradabad- 244 102, Uttar Pradesh, India

Keywords:

Gastric residence time, Floating Drug Delivery System, In-vitro evaluation

Correspondence to Author:

Juhi Dubey

Assistant Professor, Department of Pharmaceutics, IFTM University, Delhi Road, Lodhipur Rajput, Moradabad-244001, Uttar Pradesh, India

E-mail:- jdiftm@yahoo.com
ABSTRACT: The purpose of writing this review on floating drug delivery systems (FDDS) was to compile the recent literature with special focus on the types of floating drug delivery systems, principal and mechanism of floatation to achieve gastric retention. Drug delivery systems are those that float immediately upon contact with gastric fluids present promising approaches for increasing the bioavailability of drugs with absorption windows in the upper small intestine. However, immediate floating can only be achieved if the density of the device is low at the very beginning. Devices with an initially high density (which decreases with time) first settle down in the stomach and, thus, undergo the risk of premature emptying. Inherent low density can, for example, be provided by the entrapment of air or by the incorporation of low density materials or foam powder. This review explains briefly about factors affecting floating system, evaluation parameters and application of the system.

\section{INTRODUCTION: Despite tremendous advancement in drug delivery, oral route of administration has received the more attention and success because the gastrointestinal physiology offers more flexibility in dosage form design than other routes. Hence, research continuously keeps on searching for ways to deliver drugs over an extended period of time, with a well-controlled release profile.}

Gastric emptying of dosage form is extremely variable process and ability to prolong and control the emptying time. Gastric transit time is valuable asset for dosage forms, which reside in the stomach for a long period of time than conventional dosage form.

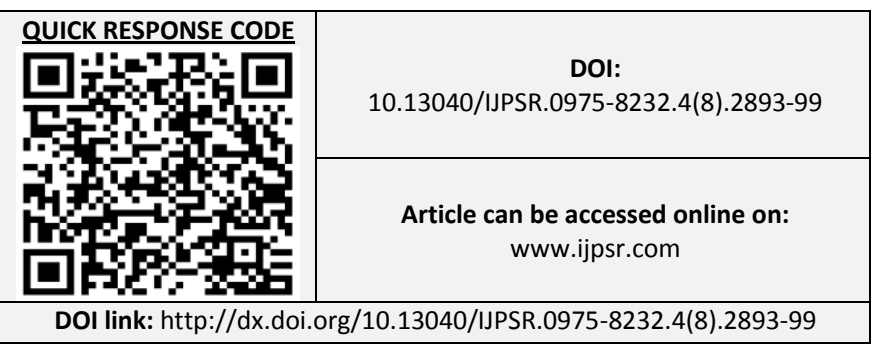

Conventional oral dosage forms such as tablets, capsules provide specific drug concentration in systemic circulation without offering any control over drug delivery and also cause great fluctuations in plasma drug levels. Many attempts have been made to develop sustained release preparations with extended clinical effects and reduced dosing frequency ${ }^{1,2}$. A problem frequently encountered with conventional sustained release dosage forms is the inability to increase their residence time in stomach and no control over drug delivery, leading to fluctuations in plasma drug level.

Gastric emptying of dosage forms is an extremely variable process and ability to prolong and control the emptying time is a valuable asset for dosage forms, which reside in the stomach for a longer period of time than conventional dosage forms. Gastric emptying occurs during fasting as well as fed states. The pattern of motility is however distinct in the 2 states. During the fasting state an inter-digestive series of electrical events take place, which cycle both through stomach and intestine every 2 to 3 hours ${ }^{3}$. 
This is called the inter-digestive myloelectric cycle or migrating myloelectric cycle (MMC), which is further divided into following 4 phases as described by Wilson and Washington ${ }^{4,5}$.

- Phase I (basal phase) lasts from 40 to 60 minutes with rare contractions.

- Phase II (preburst phase) lasts for 40 to 60 minutes with intermittent action potential and contractions.

As the phase progresses the intensity and frequency also increases gradually.

- Phase III (burst phase) lasts for 4 to 6 minutes. It includes intense and regular contractions for short period. It is due to this wave that all the undigested material is swept out of the stomach down to the small intestine. It is also known as the housekeeper wave.

- Phase IV lasts for 0 to 5 minutes and occurs between phases III and I of 2 consecutive cycles.

After the ingestion of a mixed meal, the pattern of contractions changes from fasted to that of fed state. This is also known as digestive motility pattern and comprises continuous contractions as in phase II of fasted state. These contractions result in reducing the size of food particles (to less than 1 $\mathrm{mm}$ ), which are propelled toward the pylorus in a suspension form. During the fed state onset of MMC is delayed resulting in slowdown of gastric emptying rate ${ }^{6,7}$.

Floating drug delivery systems: Floating systems are low density systems that have sufficient buoyancy to float over the gastric contents and remain in the stomach for a prolonged period. While the system floats over the gastric contents, the drug is released slowly at the desired rate, which results in increased gastro-retention time and reduces fluctuation.

(A) Non-effervescent systems: This type of system, after swallowing, swells via imbibition of gastric fluid to an extent that it prevents their exit from the stomach. The formulation methods of such type dosage forms involves the mixing of the drug with a gel, which swells when comes in contact with gastric fluid and maintains a relative integrity of shape and a bulk density of less than one within the outer gelatinous barrier. The air trapped by the swollen polymer provides buoyancy these dosage forms. The most commonly used excipients in these systems include hydroxypropyl methyl cellulose (HPMC), polyacrylate polymers, polyvinyl acetate, carbopol agar, sodium alginate, calcium chloride, polyethylene oxide and polycarbonates. This system can be further divided into four sub-types ${ }^{8,9,10}$ :

(i) Colloidal gel barrier system: These types of systems contain drug with gel-forming hydrocolloids which allow them to remain buoyant on the stomach content. This prolongs GRT and maximizes the amount of drug at its absorption sites in the solution form for ready absorption. This system incorporates a high level of one or more gelforming highly soluble cellulose type hydrocolloid as hydroxypropyl cellulose, hydroxyethyl cellulose. This hydrocolloid hydrates and forms a colloid gel barrier around its surface after coming in contact with gastric fluid and also helps in sustain releasing of drug.

(ii) Microporous Compartment system: In this technology, a drug reservoir is encapsulated inside a microporous compartment with pores along its top and bottom walls. The peripheral walls of the drug reservoir compartment are completely sealed. This sealing prevents any direct contact of gastric surface with the un-dissolved drug. The flotation chamber containing the delivery system to float over the gastric content entrapped air allows, in the stomach. Gastric fluid enters through an aperture, dissolves the drug and carries the dissolved drug for continuous transport across the intestine for absorption.

(iii) Alginate beads: To develop Multi-unit floating dosage forms, the freeze dried calcium alginate has been used. Spherical beads of approximately $2.5 \mathrm{~mm}$ in diameter can be prepared by the precipitation of calcium alginate via dropping sodium alginate solution into aqueous solution of calcium chloride. The beads are then 
separated, snap-frozen in liquid nitrogen, and freeze-dried at $-40^{\circ} \mathrm{C}$ for 24 hours, it leads to the formation of a porous system which can maintain a floating force for over 12 hours. These floating beads prolonged residence time for more than 5.5 hours.

(iv) Hollow Microspheres/Microballons: A novel emulsion solvent diffusion method used to prepare hollow microspheres loaded with drug in their outer polymer shelf ethanol/ dichloromethane solution of the drug and an enteric acrylic polymer was poured into an agitated solution of poly vinyl alcohol (PVA) that was thermally controlled at $40^{\circ} \mathrm{C}$. The gas phase is generated in the dispersed polymer droplet by the evaporation of dichloromethane formed in the internal cavity of microsphere of the polymer and drug. The microballoon floated continuously over the surface of an acidic dissolution media containing surfactant for more than $12 \mathrm{~h}$.

(B) Effervescent Systems: These buoyant systems utilize matrices prepared with swellable polymers such as methocel polysaccharides (e.g., chitosan) and effervescent components (e.g., sodium bicarbonate, citric acid or tartaric acid). The system is so prepared that when it arrives in the stomach carbon dioxide is released, causing the formulation to float in the stomach ${ }^{11}$.

\section{Advantages of Floating Drug Delivery:}

1. Enhanced Bioavailability: The bioavailability of some drugs (e.g. riboflavin and levodopa) CR-GRDF is significantly enhanced in comparison to administration of non-GRDF CR polymeric formulations 10

2. Enhanced First-Pass Biotransformation: When the drug is presented to the metabolic enzymes (cytochrome P-450, in particular CYP-3A4) in a sustained manner, the presystemic metabolism of the tested compound may be considerably increased rather than by a bolus input.

\section{Sustained drug delivery/reduced} frequency of Dosing: The drugs having short biological half-life, a sustained and slow input from FDDS may result in a flipflop pharmacokinetics and it reduces the dose frequency. This feature is associated with improved patient compliance and thus improves the therapy.

4. Targeted therapy for local ailments in the upper GIT: The prolonged and sustained administration of the drug from FDDS to the stomach may be useful for local therapy in the stomach.

5. Reduced fluctuations of Drug concentration: The fluctuations in plasma drug concentration are minimized, and concentration-dependent adverse effects that are associated with peak concentrations can be prevented. This feature is of special importance for drugs with a narrow therapeutic index that makes it possible to obtain certain selectivity in the elicited pharmacological effect of drugs that activate different types of receptors at different concentrations.

6. Reduced counter-activity of the Body: Slow release of the drug into the body minimizes the counter activity leading to higher drug efficiency.

7. Extended time over Critical (effective) concentration: The sustained mode of administration enables extension of the time

8. Improved Receptor activation selectivity: FDDS reduces the drug concentration fluctuation over a critical concentration and thus enhances the pharmacological effects and improves the clinical outcomes.

9. Minimized adverse activity at the Colon: Retention of the drug in GRDF at stomach minimizes the amount of drugs that reaches the colon and hence prevents the degradation of drug that degraded in the colon.

10. Site specific Drug Delivery: A floating dosage form is a widely accepted approach especially for drugs which have limited absorption sites in upper small intestine ${ }^{12}$, 13 . 


\section{Limitations/Disadvantages:}

i. These systems require a high level of fluid in the stomach for drug delivery to float and work efficiently-coat.

ii. Not suitable for drugs that have solubility or stability problem in GIT.

iii. Drugs such as Nifedipine which is well absorbed along the entire GIT and which undergoes first pass metabolism, may not be desirable.

iv. Drugs which are irritant to gastric mucosa are also not desirable or suitable.

v. The drug substances that are unstable in the acidic environment of the stomach are not suitable candidates to be incorporated in the systems.

vi. The dosage form should be administered with a full glass of water $(200-250 \mathrm{ml})$.

vii. These systems do not offer significant advantages over the conventional dosage forms for drugs, which are absorbed throughout the gastrointestinal tract ${ }^{14,15,16}$.

Mechanism of floating systems: Floating drug delivery systems (FDDS) have a bulk density less than gastric fluids and so remain buoyant in the stomach without affecting the gastric emptying rate for a prolonged period of time. While the system is floating on the gastric contents (Fig.: 1) the drug is released slowly at the desired rate from the system. After release of drug, the residual system is emptied from the stomach. This results in an increased GRT and a better control of the fluctuations in plasma drug concentration.

However, besides a minimal gastric content needed to allow the proper achievement of the buoyancy retention principle, a minimal level of floating force $(F)$ is also required to keep the dosage form reliably buoyant on the surface of the meal. To measure the floating force kinetics, a novel apparatus for determination of resultant weight has been reported in the literature. The apparatus operates by measuring continuously the force equivalent to $\mathrm{F}$ (as a function of time) that is required to maintain the submerged object.

The object floats better if $\mathrm{F}$ is on the higher positive side. This apparatus helps in optimizing FDDS with respect to stability and durability of floating forces produced in order to prevent the drawbacks of unforeseeable intra-gastric buoyancy capability variations ${ }^{17}$.

$\mathrm{F}=\mathrm{F}_{\text {buoyancy }}-\mathrm{F}_{\text {gravity }}=(\mathrm{Df}-\mathrm{Ds}) \mathrm{gV}$

Where, $\mathrm{F}=$ total vertical force, $\mathrm{Df}=$ fluid density, Ds $=$ object density, $\mathrm{V}=$ volume and $\mathrm{g}=$ acceleration due to gravity.

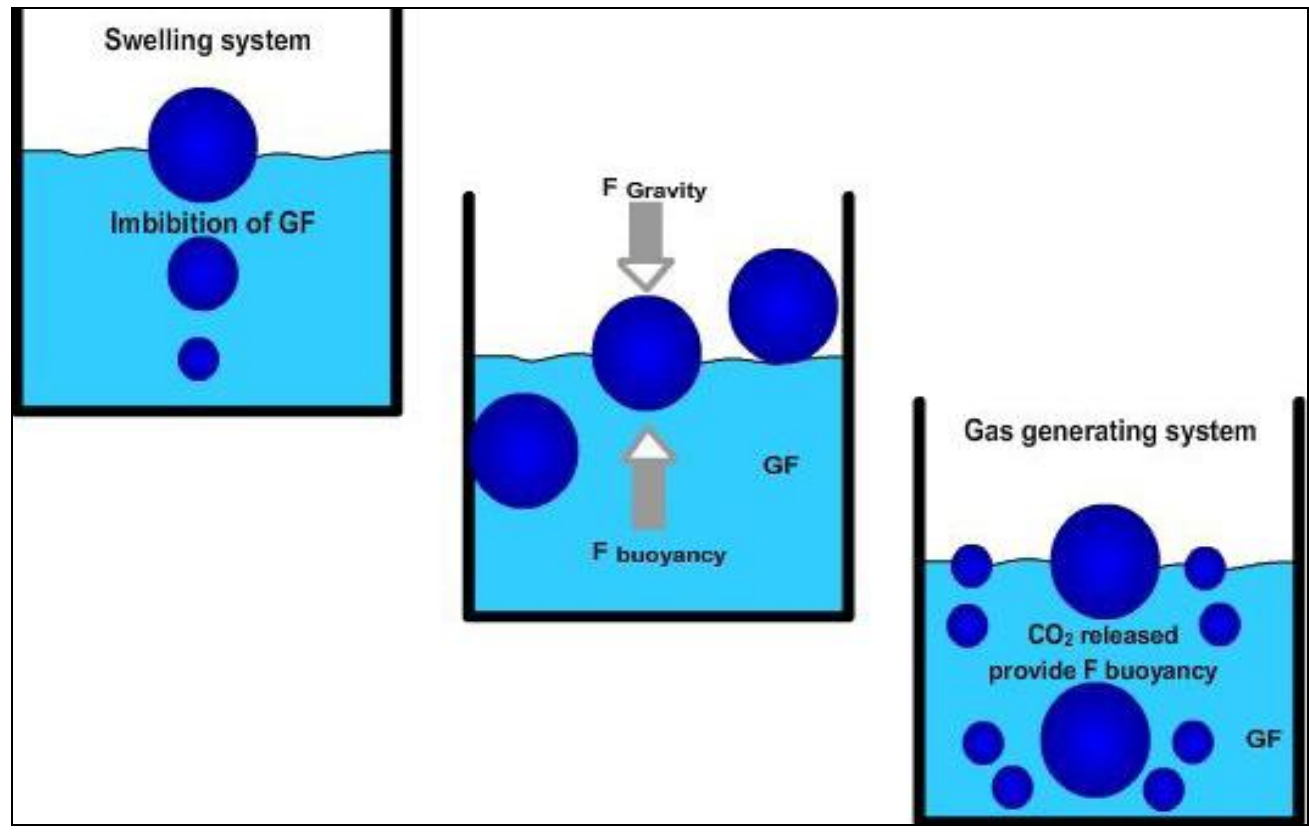

FIGURE 1: MECHANISM OF FLOATING DRUG DELIVERY SYSTEM 


\section{Drug Candidates Suitable for FDDS ${ }^{18,19,20}$ :}

a. Drugs that have narrow absorption window in GIT (e.g. L-DOPA, paminobenzoic acid, furosemide, riboflavin).

b. Drugs those are locally active in the stomach (e.g. misroprostol, antacids).

c. Drugs those are unstable in the intestinal or colonic environment (e.g. captopril, ranitidine $\mathrm{HCl}$, metronidazole).

d. Drugs that disturb normal colonic microbes (e.g. antibiotics used for the eradication of Helicobacter pylori, such as tetracycline, clarithromycin, amoxicillin).

e. Drugs that exhibit low solubility at high $\mathrm{pH}$ values (e.g. diazepam, chlordiazepoxide, verapamil).

\section{Factors affecting Floating Drug Delivery System:}

a) Density: Density of the dosage form should be less than the gastric contents $(1.004 \mathrm{gm} / \mathrm{ml})$.

b) Size and Shape: Dosage form unit with a diameter of more than $7.5 \mathrm{~mm}$ are reported to have an increased GRT competed to with those with a diameter of $9.9 \mathrm{~mm}$. The dosage form with a shape tetrahedron and ring shape devises with a flexural modulus of 48 and 22.5 kilo-pond per square inch (KSI) are reported to have better GIT for 90 to $100 \%$ retention at 24 hours compared with other shapes.

c) Fed or Unfed State: Under fasting conditions, the GI motility is characterized by periods of strong motor activity or the migrating myoelectric complexes (MMC) that occurs every 1.5 to 2 hours. The MMC sweeps undigested material from the stomach and if the timing of administration of the formulation coincides with that of the MMC, the GRT of the unit can be expected to be very short. However, in the fed state, MMC is delayed and GRT is considerably longer.

d) Nature of the Meal: Feeding of indigestible polymers of fatty acid salts can change the motility pattern of the stomach to a fed state, thus decreasing the gastric emptying rate and prolonging the drug release. e) Caloric Content: GRT can be increased between 4 to 10 hours with a meal that is high in proteins ${ }^{21,22}$.

\section{Evaluation Parameters:}

1. Size and Shape Evaluation: The particle size and shape plays a major role in determining solubility rate of the drugs and thus potentially its bioavailability. The particle size of the formulation was determined using Sieve analysis (Jayant, Mumbai), Air elutriation (Bahco TM) analysis, Photo analysis, Optical microscope (Olympus, India, Pvt. Ltd), Electro résistance counting methods (Coulter counter), Sedimentation techniques, Laser diffraction methods, ultrasound attenuation spectroscopy, Air Pollution Emissions Measurements etc.

2. Floating Properties: Effect of formulation variables on the floating properties of gastric floating drug delivery system was determined by using continuous floating monitoring system and statistical experimental design.

3. Surface Topography: The surface topography and structures were determined using scanning electron microscope (SEM, JEOL JSM - 6701 $\mathrm{F}$, Japan) operated with an acceleration voltage of 10k.v, Contact angle meter, Atomic Force Microscopy (AFM), Contact profilio-meter.

4. Swelling Studies: Swelling studies were performed to calculate molecular parameters of swollen polymers. Swelling studies was determined by using Dissolution apparatus, optical microscopy and other sophisticated techniques which include ${ }^{1} \mathrm{HNMR}$ imaging, Confocal laser scanning micro- and fats scopy (CLSM), Cryogenic Scanning Electron Microscopy (Cryo-SEM), Light scattering imaging (LSI) etc. The swelling studies by using Dissolution apparatus (USP dissolution apparatus (usp-24) Lab-India Disso 2000) was calculated as per the following formula.

Swelling ratio $=$ Weight of wet formulation / Weight of formulations

5. Determination of the Drug Content: Percentage drug content provides how much amount of the drug that was present in the formulation. 
It should not exceed the limits acquired by the standard monographs. Drug content was determined by using HPLC, HPTLC methods, near infrared spectroscopy (NIRS), Microtitrimetric methods, Inductively Coupled Plasma Atomic Emission Spectrometer (ICPAES) and also by using spectroscopy techniques (Elico Limited, Hyderabad).

6. Percentage Entrapment Efficiency: Percentage entrapment efficiency was reliable for quantifying the phase distribution of drug in the pre-pared formulations. Entrapment efficiency was deter-mined by using three methods such as Micro dialysis method, Ultra centrifugation, and pressure Ultra filtration.

7. In-vitro Release Studies: In vitro release studies (USP dissolution apparatus LABINDIA Dissolution 2000) were performed to provide the amount of the drug that is released at a definite time period. Release studies were performed by using Franz diffusion cell system and synthetic membrane as well as different types of dissolution apparatus.

8. Fourier Transforms Infrared Analysis: Fourier transform infrared spectroscopy (FTIR, Shimadzu, Model-RT-IR-8300) is a technique mostly used to identify organic, polymeric, and some inorganic materials as well as for functional group determination. Fourier Transform Infrared Analysis (FTIR) measurements of pure drug, polymer and drugloaded polymer formulations were obtained on FTIR. The pellets were prepared on $\mathrm{KBr}$-press under hydraulic pressure of $150 \mathrm{~kg} / \mathrm{cm}^{2}$; the spectra were scanned over the wave number range of 3600 to $400 \mathrm{~cm}^{-1}$ at the ambient temperature.

9. Differential Scanning Calorimetry (DSC): Shimadzu, Model-DSC-60/DSC-50/ Metler Toldeo are generally used to characterize water of hydration of pharmaceuticals. Thermograms of formulated preparations were obtained using DSC instrument equipped with an intercooler. Indium/Zinc standards were used to calibrate the DSC temperature and enthalpy scale. The sample preparations were hermitically sealed in an aluminum pan and heated at a constant rate of $10^{\circ} \mathrm{C} / \mathrm{min}$; over a temperature range of $25^{\circ} \mathrm{C}-65^{\circ} \mathrm{C}$.
Inert atmosphere was maintained by purging nitrogen gas at the flow rate of $50 \mathrm{ml} / \mathrm{min}^{23,24}$, 25.

Application of Floating Drug Delivery Systems: Floating drug delivery offers several applications for drugs having poor bioavailability because of the narrow absorption window in the upper part of the gastrointestinal tract. It retains the dosage form at the site of absorption and thus enhances the bioavailability. These are summarized as follows ${ }^{26}$, 27.

1. Sustained Release Drug Delivery System: HBS systems can remain in the stomach for long periods and, hence can release the drug over a prolonged period of time. The problem of short gastric residence time encountered with an oral CR formulation hence can be overcome with these systems. These systems have a bulk density of $<1$ as a result of which they can float on the gastric contents. These systems are relatively large in size and passing from the pyloric opening is prohibited e.g. Sustained release floating capsules of nicardipine hydrochloride were developed and were evaluated in vivo.

The formulation compared with commercially available MICARD capsules using rabbits. Plasma concentration time curves showed a longer duration for administration (16 hours) in the sustained release floating capsules as compared with conventional MICARD capsules ( 8 hours) ${ }^{28}$.

2. Site-specific drug delivery: These systems are particularly advantageous for drugs that are specifically absorbed from stomach or the proximal part of the small intestine, e.g. riboflavin and furosemide e.g. Furosemide is primarily absorbed from the stomach followed by the duodenum. It has been reported that a monolithic floating dosage form with prolonged gastric residence time was developed and the bioavailability was increased. AUC obtained with the floating tablets was approximately 1.8 times those of conventional furosemide tablets ${ }^{29}$.

3. Absorption Enhancement: Drugs that have poor bioavailability because of site specific absorption from the upper part of the 
gastrointestinal tract are potential candidates to be formulated as floating drug delivery systems, thereby maximizing their absorption e.g. a significant increase in the bioavailability of floating dosage forms (42.9\%) could be achieved as compared with commercially available LASIX tablets $(33.4 \%)$ and enteric coated LASIX-long product $(29.5 \%)^{30,31}$.

CONCLUSION: The floating drug delivery system was prepared in an effort increase the gastric retention time of the dosage form and to control drug release. One of the most feasible approaches for achieving a prolonged and predictable dug delivery profiles in the gastrointestinal tract is to control the gastric residence time, using gastro-retentive dosage forms that will provide us with new and important therapeutic options. Floating matrix tablets are designed to prolong the gastric residence time after oral administration, at a particular site and controlling the release of drug especially useful for achieving controlled plasma level as well as improving bioavailability. Although there are number of difficulties to be worked out to achieve prolonged gastric retention, a large number of companies are focusing toward commercializing this technique.

ACKNOWLEDGEMENT: The authors are thankful to Dr. RM Dubey, Vice-Chancellor, IFTM University, Moradabad, India, for making available the required facilities for this work.

\section{REFERENCES:}

1. Chikhalikar SS and Wakade RB: Floating Drug Delivery System - An Approach To Oral Controlled Drug Delivery. International Journal of PharmTech Research 2012; 4(4) 1812-26.

2. Tripathi GK and Singh S: Formulation and In vitro evaluation of $\mathrm{pH}$ sensitive oil entrapped polymeric blended buoyant beads of Amoxicillin. Scholars Research Library 2010; 2 (2): 131-38.

3. Burns SJ, Attwood D and Barnwell SG: Assessment of a dissolution vessel designed for use with floating and erodible dosage forms. International Journal of Pharmaceutics 1998; 160: 213-18.

4. Baumgartner S, Kristl J, Franc V, Vodopivec P and Zorko B: Optimisation of floating matrix tablets and evaluation of their gastric residence time. International Journal of Pharmaceutics 2000; 195: 125-35.

5. Kharia AA, Hiremath SN, Singhai AK and Jain SK: Design and optimization of floating drug delivery system of acyclovir. Indian Journal of Pharmaceutical Sciences 2010; 72(5): 599-06.

6. Bhowmik D, Chiranjib B, Margret C, Jayakar B and Kumar KPS: Floating Drug Delivery System-A Review. Scholars Research Library 2009; 1(2): $199-18$
7. Arora S: Floating drug delivery system: A review. AAPS Pharma Science Technology 2005; 6(3): 525-28.

8. Dolas RT, Hosmani A, Bhandari A, Kumar B and Somvanshi S: Nove Sustained Release Gastroretentive Drug Delivery System: A Review. International Journal of Pharma Research and Development 2011; 2(1): 2641.

9. Chretien C, Boudy V, Allain P and Chaumei JC: Indomethacin release from ion-exchange microspheres: impregnation with alginate reduces release rate. Journal of Controlled Release 2004; 96: 369-78.

10. Mathur P, Verma N: Floating drug delivery system: An innovative acceptable approach in gastroretentive drug delivery. Scholars Research Library 2010; 2(2): 257-70.

11. Hardenia SS, Jain A, Patel R and Kaushal A: Floating Drug Delivery Systems: A Review. Asian Journal of Pharmacy and Life Science 2011; 1(3): 284-93.

12. Chandel A, Chauhan K, Parashar B, Kumar H and Arora S: Floating drug delivery systems: A better approach. International Current Pharmaceutical Journal 2012; 1(5): 110-18

13. Shah SH, Patel JK, Patel NV: Stomach specific floating drug delivery system: A review. International Journal of Pharmaceutical Technology and Research 2009; 1(3): 623-33.

14. Gopalakrishnan S, Chenthilnathan A. Floating drug delivery system: A review. Journal of Pharmaceutical Science and Technology 2011; 3(2): 54854.

15. Vedha H, Chaudhary J: The recent developments on gastric floating drug delivery system: An overview. Journal of Pharmaceutical Technology and Research 2010; 2(1); 524-34.

16. Arunachalam A andKishan GK: Floating drug delivery system: A review. International Journal of Research in Pharmaceutical Sciences 2011; 2(1): 76-83.

17. Mayavanshi AV and Gajjar SS: Floating drug delivery system to increase gastric retention of drugs: A review. Research Journal of Pharmaceutical Technology 2008; 1(4): 345-48.

18. Kamalakkannan V, Puratchikody A, Prasanth VV and Masilamani K Enhancement of Drugs Bioavailability by Floating Drug Delivery System A Review. International Journal of Drug Delivery 2011; 1: 558-70.

19. Suryawanshi A and Hiremath SP: Floating Drug Delivery System - A Review. American Journal of Pharmatech Research 2011; 2(1): 138-53.

20. Shubhrajit M, Thilothama LR and Shashanka D. Formulation and in vitro evaluation of metoprolol succinate floating tablets by using two viscosity grade of HPMC. International Journal of Pharmaceutical Science and Research 2012; 3 (9): 3507-13

21. Bardonnet PL, Faivre V, Pugh WJ, Piffaretti JC and Falson F: Gastroretentive Dosage Forms: Overview and Special case of Helicobacter pylori. Journal of Control Release 2006; 111: 1 - 18.

22. Narang N: AN Updated Review On: Floating Drug Delivery System (FDDS). International Journal of Applied Pharmaceutics 2011; 3(1): 1-7.

23. Chandiran S, Kumar BP and Narayan V: Formulation and in vitro evaluation of floating drug delivery system for salbutamol sulphate. International Journal of Pharma Biomed Sciences 2010; 1(1): 12-15.

24. Jain A: New Concept: Floating Drug Delivery System. Indian Journal of Novel Drug Delivery 2011; 3(3): 163-69.

25. Geetha A, Rajendra K, Mohan CHK, Sateesh V and Raju PN: A Review on Floating Drug Delivery Systems. International Journal of Pharmaceutical Research and Biomedical Analysis 2012; 1(1): 1-13.

26. Dwivedi S and Kumar V: Floating Drug Delivery Systems- A Concept of Gastroretention Dosages Form. International Journal of Research in Pharmaceutical and Biomedical Sciences 2011; 2(4): 1413-26.

27. Kaza R, Usharani E, Nagaraju R, Haribabu R and Reddy PVS: Design and Evaluation of Sustained release Floating tablets for the treatment of Gastric Ulcers. Journal of Pharmaceutical. Sciences \& Research 2009; 1(4): 81-87.

28. Mantry S, Thilothama LR and Shashanka D: Formulation and in vitro evaluation of Metoprolol Succinate Floating Tablets by using two viscosity grade of HPMC. International Journal of Pharmaceutical Sciences and Research 2012; 3(9): 3507-13.

29. Raju DB, Sreenivas R and Varma MM: Formulation and evaluation of floating drug delivery system of Metformin Hydrochloride. Journal of Chemical and Pharmaceutical Research 2010; 2(2): 274-78.

30. Sarojini S, Arivazagan D, Manavalan R and Jayanthi V: Buoyant Sustained Release Tablets Based On Polyethylene Oxide. International Journal of Pharmacy and Pharmaceutical Sciences 2010; 2(1): 144-49.

31. Pare A, Yadav SK and Patil UK: Formulation and Evaluation of Effervescent Floating Tablet of Amlodipine besylate. Research Journal of Pharmaceutics and Technology 2008; 1(4):526-30.

\section{How to cite this article:}

Dubey J and Verma N: Floating Drug Delivery System: A Review. Int J Pharm Sci Res 2013: 4(8); 2893-2899. doi: 10.13040/IJPSR. 0975-8232.4(8).2893-99

All $\odot 2013$ are reserved by International Journal of Pharmaceutical Sciences and Research. This Journal licensed under a Creative Commons Attribution-NonCommercial-ShareAlike 3.0 Unported License.

This article can be downloaded to ANDROID OS based mobile. Scan QR Code using Code/Bar Scanner from your mobile. (Scanners are available on Google Playstore) 